\title{
Comparative Analysis of Color Image Denoising Techniques
}

\author{
Amandeep Singh ${ }^{1}$, Gaurav Sethi ${ }^{2}$ and G.S Kalra ${ }^{3}$ \\ ${ }^{I}$ School of Electronics and Electrical Engineering, Lovely Professional University, Jalandhar, Punjab, India. \\ ${ }^{2}$ School of Electronics and Electrical Engineering, Lovely Professional University, Jalandhar, Punjab, India. \\ ${ }^{3}$ Electronics and Communication, CT Group of Institutions, Jalandhar, Punjab, India.
}

ORCID: 0000-0001-5706-760X (Amandeep Singh)

\begin{abstract}
The choice of efficient algorithms for denoising digital images is still a scientific challenge to the intersection of functional analysis, statistics and computing. There have been a few distributed calculations and each approach has its suppositions, its key operational points, and its constraints. In this paper, a brief comparative analysis of some significant work in the field of image denoising is Presented. Image denoising techniques which are suitable for color images are classified and compared by using parameter like Peak signal to noise ratio PSNR. The visual and quantitative outcomes demonstrate that the ROAD TGM filter outflanks the current filtering methods in reestablishing the original image which is deteriorated by impulse noise.
\end{abstract}

Keywords: Denoising, Salt and Pepper noise, Impulse noise, PSNR.

\section{INTRODUCTION}

Images are part of our day to day life and nowadays these have become part of our lifestyle. Applications of an image are enormous from considered from a figure print scanner to face detection and biomedical image processing. The images that are utilized in the day to day life is containing various information that can be used to identify a person or an event. So, it is important to maintain the images in their original and clear form. This is not an easy task as it is very difficult to avoid the scenarios under which noise can affect the image. In the field of digital images quality is usually distorted by the impulse noise (it is a noise which corrupts the image with peak high and peak low values). This noise occurs at the time of image acquisition and image storing (both steps cannot be avoided). That's why it becomes important to remove the noise before the image is utilized further for analysis otherwise it will lead towards misinterpretation[1]-[5]. The process of restoring the corrupted image is known as image filtration or image denoising. To measure the performance and to evaluate the operational quality of algorithm quantitative parameters are required, for this purpose various parameters are available but the most suitable or preferred parameter is Peak signal to noise ratio. As we had already discussed that images are affected by several types of noises, but in the routine practical conditions impulse noise usually affects the images and the impact of Impulse noise on the digital image is most severe in comparison to other noises[6]-[11]. Moreover, the processes of capturing the image contain steps and components like camera sensor, image quantization, signal amplification which induce disturbances and it can be discomforting for understanding and image processing. The purpose of noise filtering is to reduce intensity variations within each region of the image while respecting the integrity of scenes: transitions between homogeneous regions, significant elements of the image must be preserved for the best quality[3], [12]-[15]. This paper describes the performance of different techniques of denoising giving an insight into the mechanism to know which algorithm should be used to achieve reliable results. Further, we focus on analyzing the color images on different noises levels to identify the overall performance of algorithms. This manuscript is systematized as given: In section 2, the Salt and Pepper noise is presented. In section 3, we expose some filters classics and we present detail of filters used for comparative analysis. In section 4 dedicated to result in discussions (Denoising noise of color images).

\section{IMAGE NOISE MODELS}

At each stage of the acquisition of a scene, disturbances (scratches, dust, camera, amplification, quantification) will deteriorate the quality of the image. These disturbances are grouped under the name of "image noise"[16]. The image noise can be categorized into two categories:

$$
\begin{aligned}
& \text { - } \quad \text { Independent noise (we speak of random noise) } \\
& \text { - The noise that depends on the image data. }
\end{aligned}
$$

We can represent the noise affected image with the following expression

$$
S(a, b)=w(a, b)+z(a, b)
$$

$\mathrm{S}(\mathrm{a}, \mathrm{b})$ is defines as the combination of the real image $w(a, b)$ and noise $\mathrm{z}(\mathrm{a}, \mathrm{b})$. The noise $\mathrm{z}(\mathrm{a}, \mathrm{b})$ is frequently defined by its variance $\sigma_{n}{ }^{2}$ square. PSNR defines the quality of an image under the influence of noise reduces. Similarly, $\sigma_{w}^{2}$ and $\sigma_{y}^{2}$ represent the respective variances of the actual and the processed image. The noise of the image is considered as a random field. Because of the different mechanism are involved in image acquisition, 
the level of noise is affecting the image can be different. So, an algorithm needed which can remove this wide range of noises level. The most common noise in a digital image is Salt and Pepper noise which is also referred as shot noise, binary noise and impulse noise. This is caused while capturing and sending the images because of impairments involved in the communication process. It has just two conceivable values $(0$ and 255). The likelihood of the occurrence of any of these two vales is normally under 0.1 . The noisy pixels are kept on the other hand to the minimum value or to the most extreme value, provide the picture a "Salt and Pepper" like resemblance[2], [17]-[21]. Noise-free pixels in the affected image stay unaltered. For the image of 8-bit, the representation of Pepper noise in the digital image is done by using value zero and for Salt noise reflection is achieved by using value 255 . To represent the effect of noise on image, $10 \%$ Salt and Pepper noise affected image is shown in figure 2 (a).

\section{IMAGE DENOISING TECHNIQUES}

The image denoising techniques are required to remove the noise existing in the image. But these techniques are generally designed for gray scale images. To make these techniques compatible with color images an iterative mechanism is required to be produced which can pipeline the component of images (Red, Green and Blue image) of color image. So, by breaking the color image into 3 components a gray scale algorithm can be made compatible with them. Let us consider an example gray scale images which are having single matrix containing pixel intensity value from 0 to 255 , whereas if we break a color image into its three basic color components then one by one separately processing can be applied to them and these separate images can be combined to produce color image at the end again. Now let's discuss the algorithms considered for this comparative analysis. Let's start with first one which is ROAD (Rank Ordered Absolute Difference) this method is quite efficient when image is affected by uniform noise[22], [23]. This method is based on a window to detect the pixel affected by noise, so these detected pixels can be removed from image. Let $s=(s 1, s 2)$ be the pixel position below the threshold value and $\Omega \mathrm{s}(\mathrm{N})$ be the number of point in a $(2 \mathrm{~N}+1) \times(2 \mathrm{~N}+1)$ surrounding concentrated at $\mathrm{s}$ for creation of window.

$$
\Omega \mathrm{s}(\mathrm{N})=\{s+(i, j)-N \leq i, j \leq N
$$

Let us consider $\mathrm{N}=1$. Hence $\Omega \mathrm{z}$ denotes the set of points in a $3 \times 3$ identified surrounding of $\mathrm{s}$. Given by expression (3)

$$
\Omega \mathrm{Z}=\Omega \mathrm{s}(1) /\{\mathrm{s}\}
$$

For every one point of $\mathrm{y} \in \Omega \mathrm{z}$ define $d_{x, y}$ as the complete difference in strength of the pixel among $s$ and $y$. Absolute difference is expressed by following expression (4)

$$
d_{x, y}=\left|u_{s}-u_{y}\right|
$$

Sort the $d_{x, y}$ value in ascending order and describe the ROAD by following expression (5)

$$
R O A D_{m}(s)=\sum_{i=1}^{m} r_{i}(s) 1<i<m
$$

Where $2 \leq m \leq(2 N+1)^{2}-1$ and $r_{i}(s)$ is the smallest $d_{x, y}$ for $\mathrm{y} \in \Omega \mathrm{z}$.

Let's suppose for $\mathrm{N}=1$ and for $\mathrm{m}=4$, ROAD offers how much closeness is present in the pixel intensity value to its 4 nearest neighbors in $3 \times 3$ window. Rationale under this measurement is that noisy pixel will shift incredibly in its intensity values from maximum of its adjoining pixels so that, ROAD value would be bigger and in place of uncontaminated pixel that has a place with genuine picture are going to have large portion of the neighboring pixel of comparative intensity so ROAD value will be littler. ROAD value can be utilized to recognize a pixel undermined by noise affect by setting a specific threshold value. On the off chance that the ROAD value is more prominent than the threshold value the pixel is considered to be affected by noise. It is recommended in to utilize a $3 \times 3$ window and $m=4$ for the noise level under $25 \%$ generally $5 \times 5$ window and $m=12$.

In the Trimmed Global Mean (TGM) Filter calculation begins by the identification of noisy pixels. On the off chance that the preparing pixels assume $P(i, j)$ is in the vicinity of 0 and 1 , at that point the pixel in uncorrupted and it stays same yet in the event that the $\mathrm{P}(\mathrm{i}, \mathrm{j})$ is 0 or 1 then it is determined as noisy pixels. For noisy pixels, we choose a window of size N X N and eliminate every noise affected pixel after the chosen window, to achieve the stated elimination, it becomes important to locate the median of the rest of the pixels and replace the noise affected pixels with the median value. In the event that they chose window contains whole pixels are noisy pixels at that point noisy pixel is supplanted by trimmed global mean. Trimmed global mean is figured by removing all the undermined pixels from the entire picture and calculating the mean of the rest of the pixels.

It is a two-phase calculation, in the primary stage the noisy pixels in the image applied for denoising is distinguished utilizing rank order absolute difference (ROAD) algorithm. In the subsequent phase, the degraded pixels are replaced by the median of the noiseless pixels in the chosen window. Trimmed global mean filter is utilized, if the chosen window comprises all of the pixels as noise affected pixels. Then TGM is calculated by eliminating the noise corrupted. pixels from the window and mean of the uncorrupted pixels is obtained to replace the value with noisy pixel. In this manner, it doesn't take abundant handling time however still provides great outcomes for high noise density. The fundamental points of interest of the ROAD TGM calculation are that it is anything but difficult to actualize in equipment and that it has low run time[24].

DBMF (Decision Based Median Filter) was proposed to deal with impulse noise (Salt and Pepper noise) as we already know this noise exists at two value 0 or 255 . So, this algorithm checks the existence of noise from the beginning and identifies the noise locations and considers the values as original values if 
they exist between 1 and 254 and then this filter uses median value to restore the original value. Drawback of this filter is when hight level of noise in introduced in image then its effectiveness is reduced.

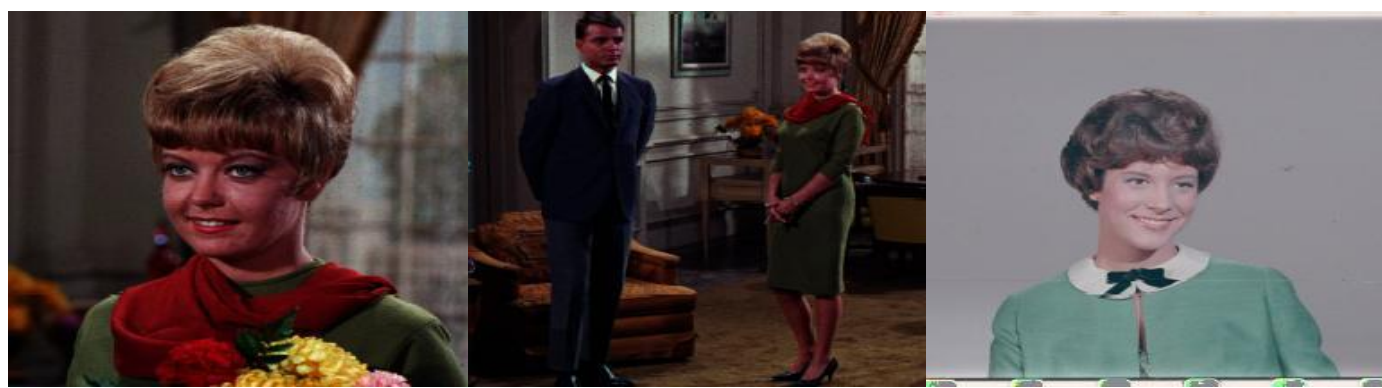

(a)

(b)

(c)

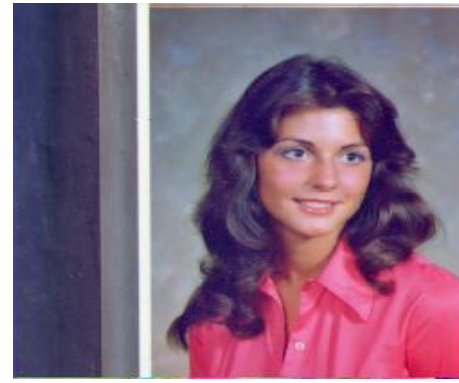

(d)

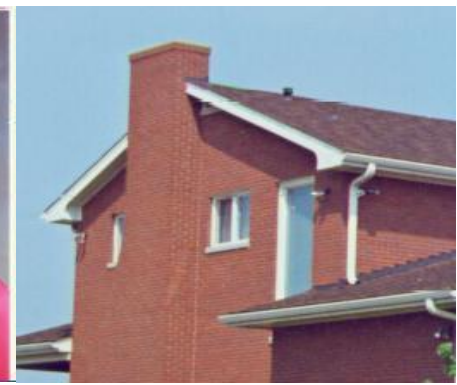

(e)

Figure 1. Original color image Data set. (a) Female-A image, (b) Couple image, (c) Female-B image, (d) Female-C image, (e) House image.

\section{RESULTS AND DISCUSSION}

For the result analysis of algorithms, the data set of color images is initially affected by Salt and Pepper noise with the increasing power of noise ranging from $10 \%$ noise to $50 \%$ noise. Five color images considered in the dataset are shown in figure 1. Let's understand the analysis process with an example of the original image Female-A shown in figure 1 (a). This image is affected by 5 stages of Salt and Pepper noise in increasing order starting from $10 \%$ then goes up to $50 \%$ $(10 \%, 20 \%, 30 \%, 40 \%$ and $50 \%)$. Noise affected images are shown in figure 2 (a), figure 3 (a),--- figure 6 (a).Corresponding filtered images produced by the algorithms (ROAD-TGM and
DBMF) are shown in figure 2 (b), figure 3 (b),--- figure 6 (b) and figure 2 (c), figure 3 (c),--- figure 6 (c) respectively. Each result shows the comparative visual representation of denoising operation performed by both algorithms on each noise percentage level. The performance of noise filtration algorithms is evaluated on the bases of visual results and a quantitative parameter PSNR. To consider an algorithm performance superior, its results should have comparatively better visual clarity of filtered image and higher PSNR value than the other algorithm. Results show that the performance of ROAD-TGM algorithm is better than DBMF algorithm for color image Female-A which can be observed from the visual comparison of images shown in the results below.

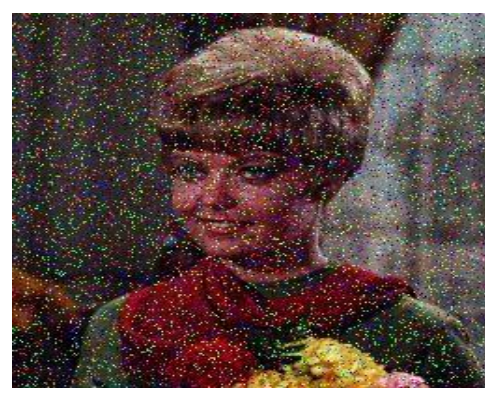

(a)

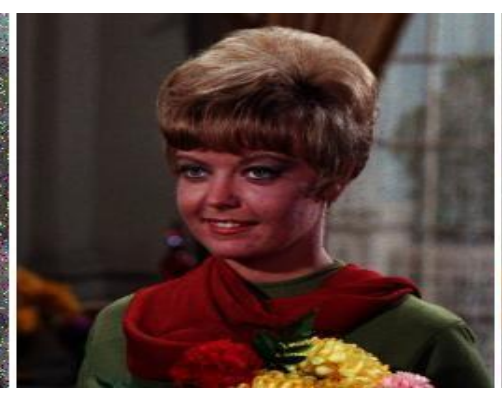

(b)

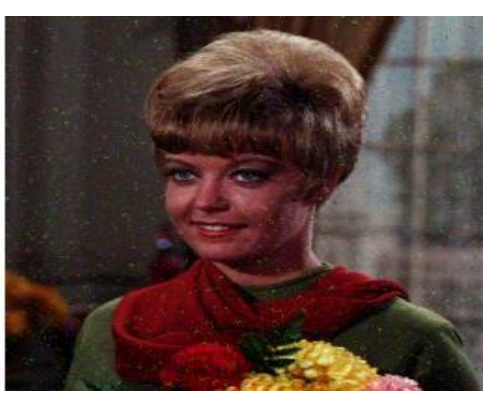

(c)

Figure 2. (a) image effected with $10 \%$ noise (b) image filtered by ROAD-TGM, (c) image filtered by DBMF. 


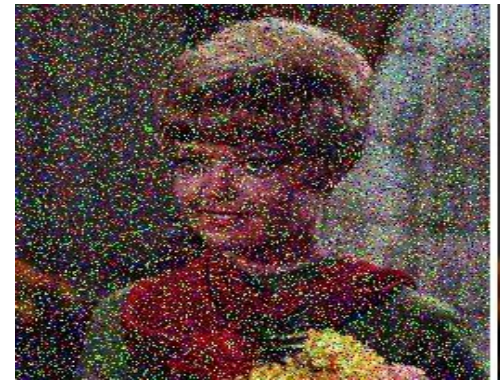

(a)

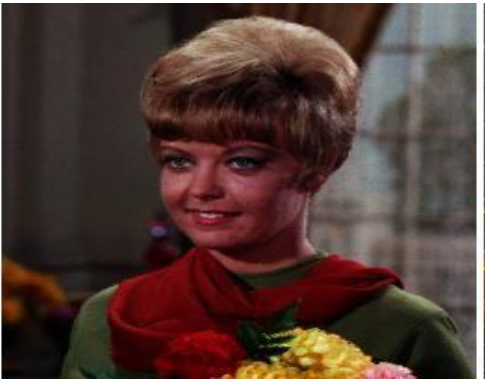

(b)

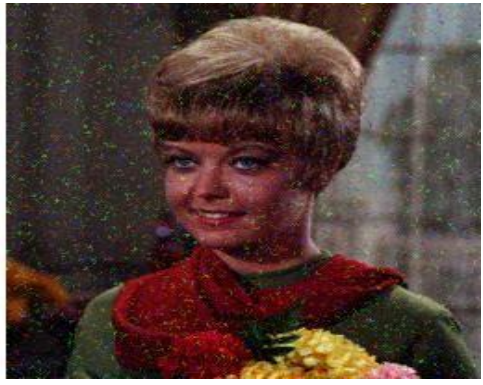

(c)

Figure 3. (a) image effected with $20 \%$ noise (b) image filtered by ROAD-TGM, (c) image filtered by DBMF.

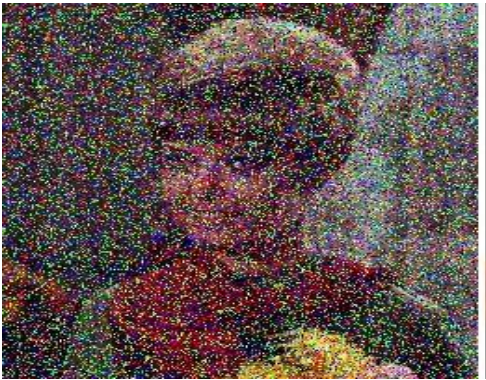

(a)

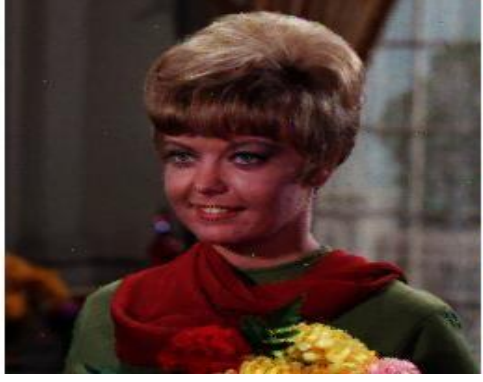

(b)

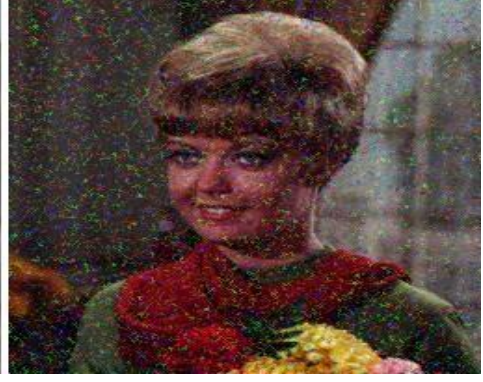

(c)

Figure 4. (a) image effected with $30 \%$ noise (b) image filtered by ROAD-TGM, (c) image filtered by DBMF.

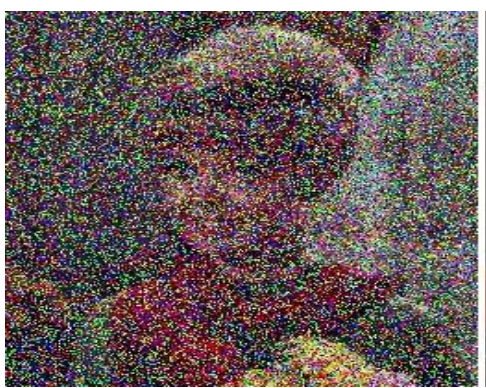

(a)

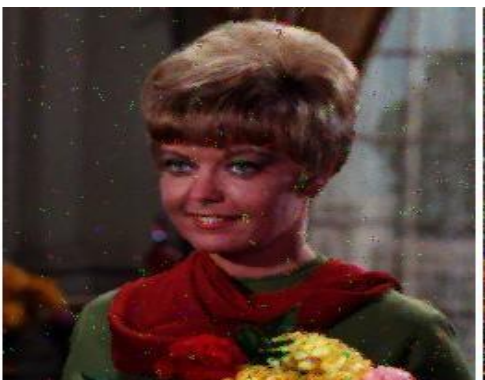

(b)

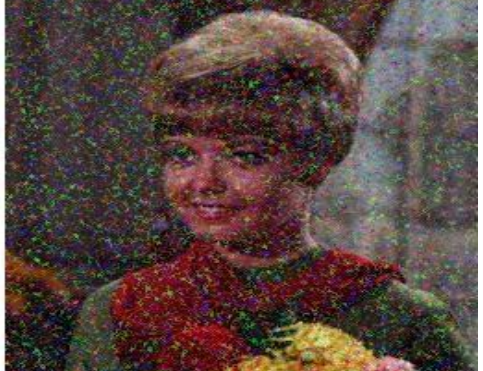

(c)

Figure 5. (a) image effected with $40 \%$ noise (b) image filtered by ROAD-TGM, (c) image filtered by DBMF.

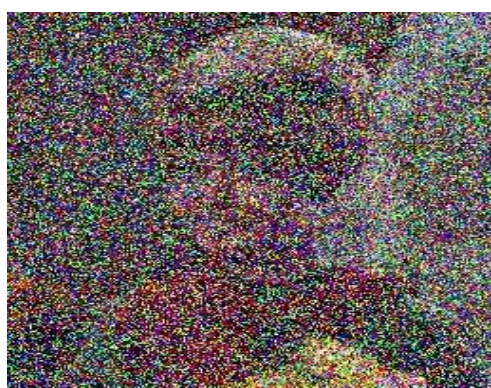

(a)

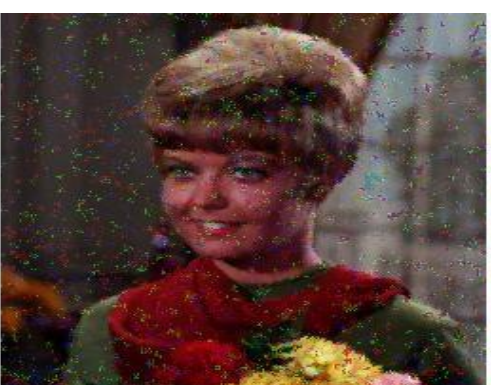

(b)

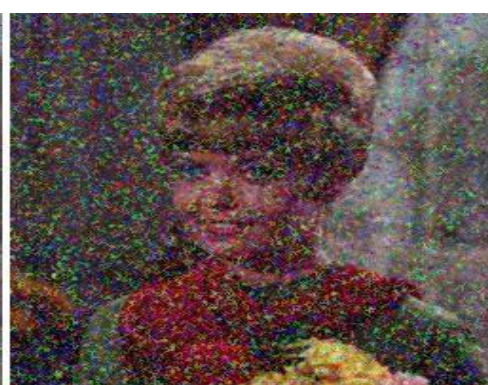

(c)

Figure 6. (a) image effected with $50 \%$ noise (b) image filtered by ROAD-TGM, (c) image filtered by DBMF.

Similarly, the results of the remaining four images of the color data set can be calculated. To clearly indicate the performance difference between these two algorithms line graphs are utilized which shows the change in PSNR values of both algorithms with respect to change in noise levels. Line graphs representing the comparative analysis of ROAD-TGM and DBMF for the entire color image data set are shown in figure 7 to figure 10. Let's understand the graphs for the female-A image of the data set shown in figure 7. PSNR value of 39.11 is achieved by denoised image produced by ROAD-TGM 
algorithm when $10 \%$ noise affected image is given as input comparison to lesser PSNR value produced by DBMF algorithm (29.44). In this manner analysis of denoised image from $20 \%, 30 \%, 40 \%$ and $50 \%$ noise affected female-A images can be observed from the figure 7. Road-TGM algorithm achieves higher PSNR value for each noise level in comparison with DBMF algorithm. Typically, PSNR value achieved by ROAD-TGM for $20 \%, 30 \%, 40 \%$ and $50 \%$ noise level are $35.11,31.96,27.50$ and 22.00 in comparison to DBMF which achieves lower values of PSNR 24.16,20.74,18.11 and 15.97 respectively. Performance of Both algorithms decay as the noise level of Salt and Pepper noise increase from low to high. This decay in PSNR value of a denoised image is more in DBMF algorithm. Similarly, the results of other images of the data set are provided in figure 8 to figure 10. In all these results of PSNR similar pattern can be observed that ROAD TGM is surpassing the performance of DBMF algorithm.

PSNR FOR FEMALE-A IMAGE

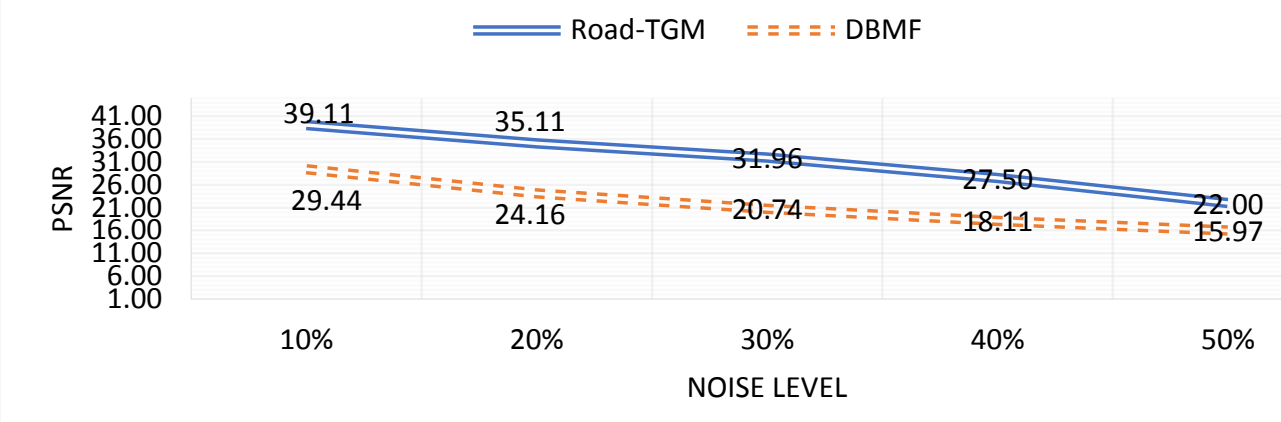

Figure 7. PSNR results for female-A image denoised by ROAD-TGM and DBMF.

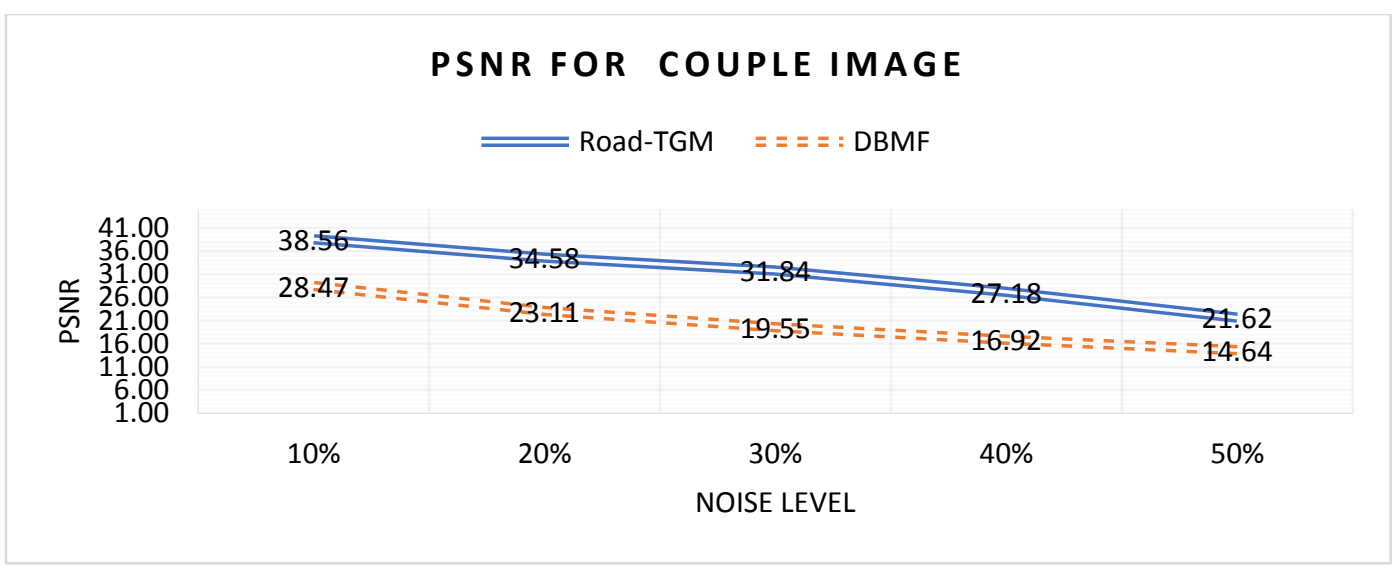

Figure 8. PSNR results for couple image denoised by ROAD-TGM and DBMF.

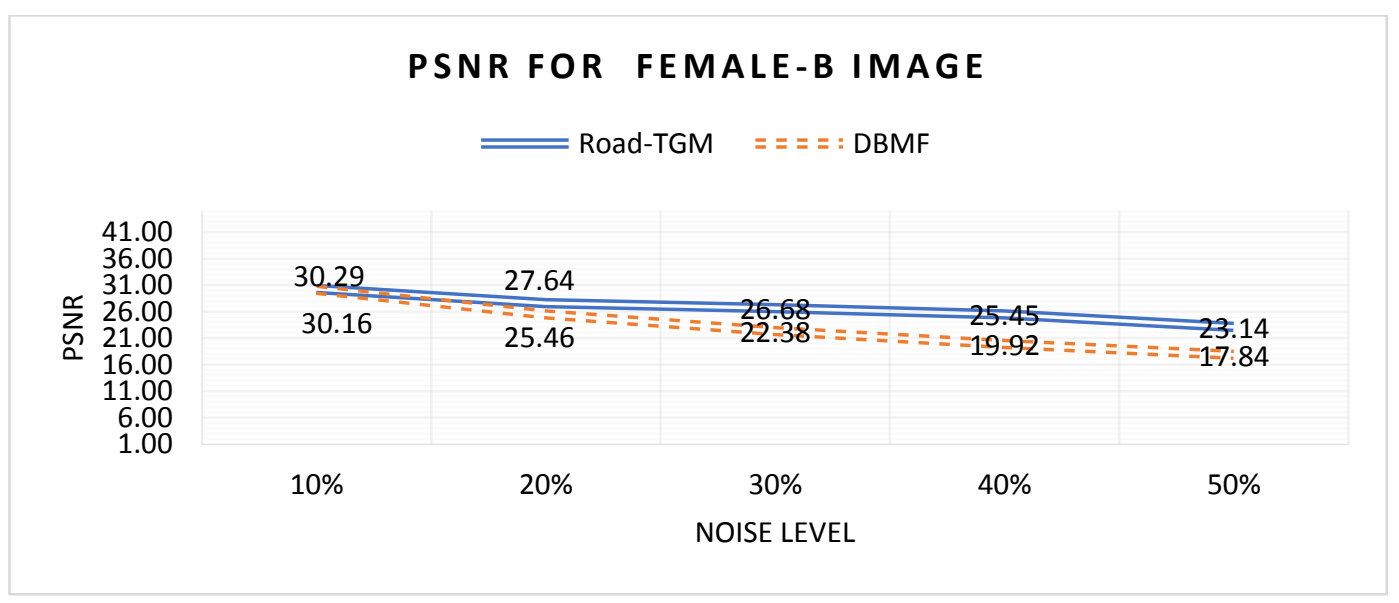

Figure 9. PSNR results for female-B image denoised by ROAD-TGM and DBMF. 


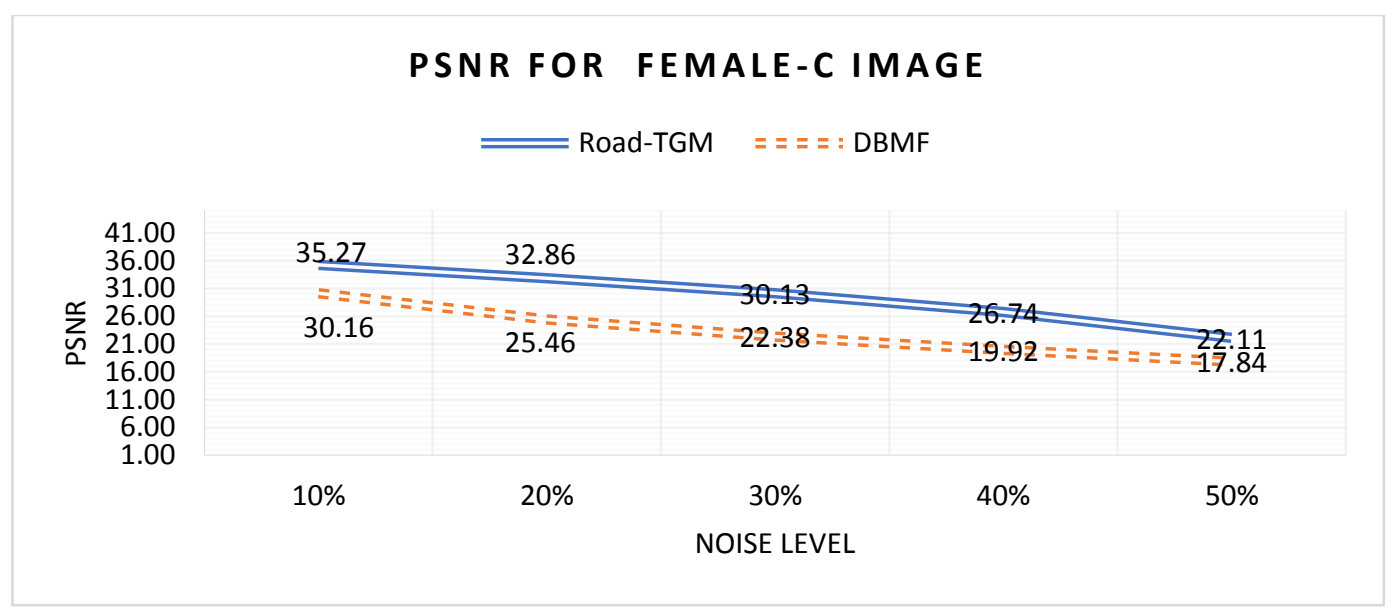

Figure 10. PSNR results for female-B image denoised by ROAD-TGM and DBMF.

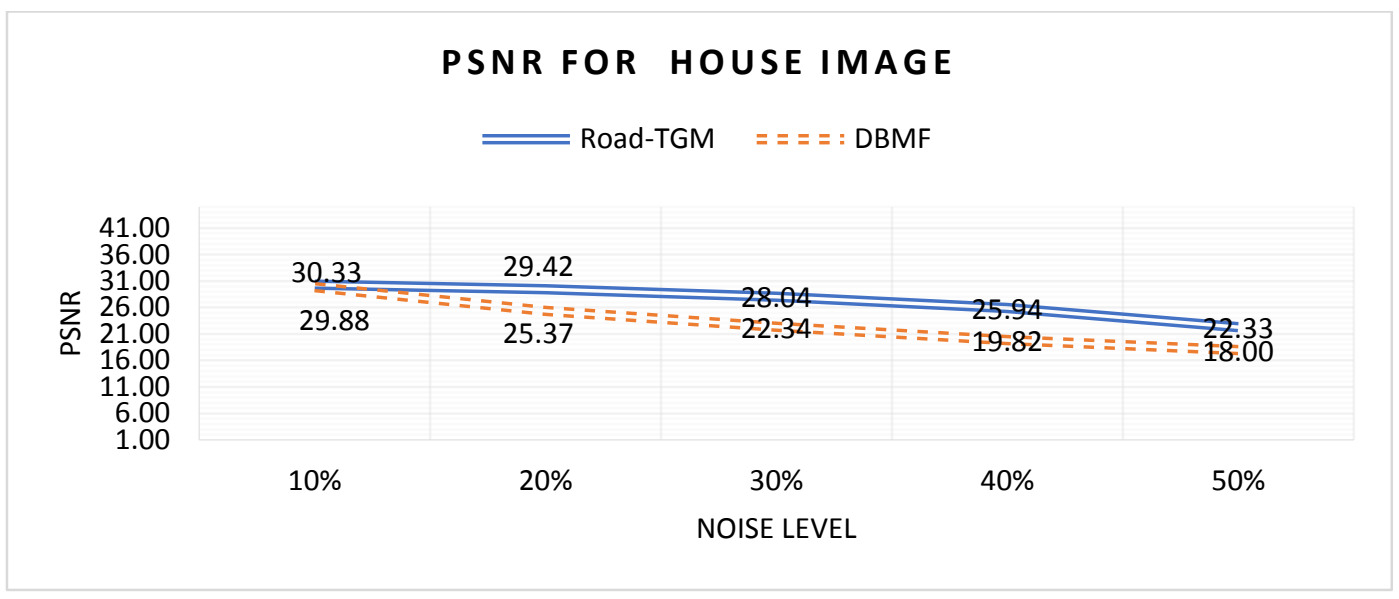

Figure 11. PSNR results for house image denoised by ROAD-TGM and DBMF.

Table 1. Comparative analysis of mean PSNR values of algorithms.

\begin{tabular}{|c|c|c|c|c|c|}
\hline Noise Level & $\mathbf{1 0 \%}$ & $\mathbf{2 0 \%}$ & $\mathbf{3 0 \%}$ & $\mathbf{4 0} \%$ & $\mathbf{5 0 \%}$ \\
\hline ROAD-TGM & 34.71 & 31.92 & 29.73 & 26.56 & 22.24 \\
\hline DBMF & 29.62 & 24.55 & 21.48 & 18.72 & 16.86 \\
\hline
\end{tabular}

To understand the overall behavior of algorithms a table of mean PSNR values is presented (Table 1). This table contains mean PSNR of five color images of data set with respect to increasing noise levels $10 \%$ to $50 \%$. This table shows that denoising capability of both algorithms decreases with an increase in noise level. But this decrement is comparatively less in ROAD-TGM. .

\section{CONCLUSION}

In this work analysis of two algorithms, ROAD-TGM and DBMF is presented for the color image data set. Data set of noise image is constructed by producing noise affected images initiating from $10 \%$ of salt and peer noise to $50 \%$ noise then these noises affected images are restored by respective denoising algorithms. As both algorithms considered in this analysis are working in the spatial domain they work on the identification of noise and then filtration of noisy pixels. As noise increases it becomes difficult for both algorithms to maintain the denoising performance. For low level of noises both algorithms work fine but as noise increase difference in the performance of both algorithms tends to have deviation. ROAD-TGM algorithm shows comparatively superior performance in all considered noise levels of Salt and Pepper noise. 
International Journal of Engineering Research and Technology. ISSN 0974-3154, Volume 13, Number 10 (2020), pp. 2761-2767

(C) International Research Publication House. https://dx.doi.org/10.37624/IJERT/13.10.2020.2761-2767

\section{REFERENCES}

[1] F. Russo, "Impulse noise cancellation in image data using a two-output nonlinear filter," Meas. J. Int. Meas. Confed., vol. 36, no. 3-4, pp. 205-213, 2004.

[2] N. K. Chaitanya and P. Sreenivasulu, "Removal of salt and pepper noise using Advanced Modified Decision based Unsymmetric Trimmed Median Filter," in 2014 International Conference on Electronics and Communication Systems, ICECS 2014, 2014.

[3] M. Nikolova, "A Variational Approach to Remove Outliers and Impulse Noise," J. Math. Imaging Vis., vol. 20, no. 1-2, pp. 99-120, 2004.

[4] J. K. Mandal and A. Sarkar, "A novel Modified Directional Weighted Median Based filter for removal of random impulse noise (MDWMF)," Proc. - 2010 Int. Symp. Electron. Syst. Des. ISED 2010, pp. 230-234, 2010 .

[5] A. Singh, G. Sethi, and G. S. Kalra, "Spatially Adaptive Image Denoising via Enhanced Noise Detection Method for Grayscale and Color Images," IEEE Access, vol. 8, pp. 112985-113002, 2020.

[6] C. T. Lu, Y. Y. Chen, L. L. Wang, and C. F. Chang, "Removal of salt-and-pepper noise in corrupted image using three-values-weighted approach with variable-size window," Pattern Recognit. Lett., vol. 80, pp. 188-199, 2016.

[7] A. Nasimudeen, M. S. Nair, and R. Tatavarti, "Directional switching median filter using boundary discriminative noise detection by elimination," Signal, Image Video Process., vol. 6, no. 4, pp. 613-624, 2012.

[8] Y. A. Vlasov and S. J. McNab, "Coupling into the slow light mode in slab-type photonic crystal waveguides," Opt. Lett., vol. 31, no. 1, p. 50, Apr. 2006.

[9] A. Buades, B. Coll, and J. M. Morel, "Image denoising methods. A new nonlocal principle," SIAM Rev., vol. 52, no. 1, pp. 113-147, 2010.

[10] G. Shikkenawis and S. K. Mitra, "2D orthogonal locality preserving projection for image denoising," IEEE Trans. Image Process., vol. 25, no. 1, pp. 262-273, 2016.

[11] I. Irum, M. Sharif, M. Raza, and S. Mohsin, “A nonlinear hybrid filter for salt \& Pepper noise removal from color images," J. Appl. Res. Technol., vol. 13, no. 1, pp. 79$85,2015$.

[12] Z. Li, Y. Cheng, K. Tang, Y. Xu, and D. Zhang, “A salt $\&$ pepper noise filter based on local and global image information," Neurocomputing, vol. 159, no. 1, pp. 172$185,2015$.

[13] M. G. Her, M. Karkoub, and J. M. Chen, "Design and application of a low cost visual tracking system," Aust. J. Electr. Electron. Eng., vol. 4, no. 2, pp. 191-200, Jan. 2008.

[14] P. Thomas, B. Price, C. Paine, and M. Richards, "Remote electronic examinations: Student experiences,"
Br. J. Educ. Technol., vol. 33, no. 5, pp. 537-549, 2002.

[15] D. Brunet, E. R. Vrscay, and Z. Wang, "On the mathematical properties of the structural similarity index," IEEE Trans. Image Process., vol. 21, no. 4, pp. 1488-1495, Apr. 2012.

[16] P. S. Windyga, "Fast impulsive noise removal," IEEE Trans. Image Process., vol. 10, no. 1, pp. 173-179, 2001.

[17] Kamarujjaman, M. Mukherjee, and M. Maitra, "A new decision-based adaptive filter for removal of high density impulse noise from digital images," in 2014 International Conference on Devices, Circuits and Communications, ICDCCom 2014 - Proceedings, 2014.

[18] M. Nasri, S. Saryazdi, and H. Nezamabadi-Pour, "SNLM: A switching non-local means filter for removal of high density salt and pepper noise," Sci. Iran., vol. 20, no. 3, pp. 760-764, 2013.

[19] H. R. Sheikh and A. C. Bovik, "Image information and visual quality," IEEE Trans. Image Process., vol. 15, no. 2, pp. 430-444, Feb. 2006.

[20] M. S. Nair and G. Raju, "A new fuzzy-based decision algorithm for high-density impulse noise removal," Signal, Image Video Process., vol. 6, no. 4, pp. 579-595, 2012.

[21] S. Esakkirajan, T. Veerakumar, A. N. Subramanyam, and C. H. PremChand, "Removal of High Density Salt and Pepper Noise Through Modified Decision Based Unsymmetric Trimmed Median Filter," IEEE Signal Process. Lett., vol. 18, no. 5, pp. 287-290, 2011.

[22] V. Patanavijit and K. Thakulsukanant, "Performance Inspection of Denoising Method Implemented by Decision Based Adaptive Median Filter for Using under RVIN Condition," in Proceedings - 2019 19th International Symposium on Communications and Information Technologies, ISCIT 2019, 2019, pp. 422 425.

[23] I. Aizenberg and C. Butakoff, "Effective Impulse Detector Based on Rank-Order Criteria," IEEE Signal Process. Lett., vol. 11, no. 3, pp. 363-366, 2004.

[24] G. S. Kalra and S. Singh, "Efficient digital image denoising for gray scale images," Multimed. Tools Appl., vol. 75, no. 8, pp. 4467-4484, 2016. 